\title{
Sea Ice Dynamics Induced by External Stochastic Fluctuations
}

\author{
Dmitri V. Alexandrov, ${ }^{1}$ Irina A. Bashirirtseva, ${ }^{1}$ Alexey P. Malygin, ${ }^{1}$ and Lev B. Ryashioo ${ }^{1}$
}

\begin{abstract}
The influence of stochastic fluctuations in the atmosphere and in the ocean caused by different occasional phenomena (noises) on dynamic processes of sea ice growth with a mushy layer is studied. It is shown that atmospheric temperature variances substantially increase the sea ice thickness, whereas dispersion variations of turbulent flows in the ocean to a great extent decrease the ice content produced by false bottom evolution.
\end{abstract}

Key words: Sea ice, mushy layer, false bottom, stochastic fluctuations, nonlinear dynamics.

\section{Introduction}

Recent studies of the ice cover changes have refocused attention on the correct description of local processes that have large scale consequences. For instance, the growth and decay of sea ice in the Polar Regions is the high-latitude equivalent of the evaporation-precipitation cycle in the remainder of the world's oceans (AAGARD and CARMACK, 1994). One of the important contributions of the ice evolution is connected with cracks in the perennial ice cover, known as leads. These transient fissures attract scientific attention because they provide a thermal conduit through which heat- and radiative-transfer processes are enhanced tremendously, relative to the thick pack ice that surrounds them. The thermal importance of leads was put in context by BADGLEY (1966), who showed that during winter the atmospheric heat flux from rapidly freezing leads can be several orders of magnitude larger than over perennial sea ice. He emphasized the large-scale implications of leads by arguing that they need

1 Department of Mathematical Physics, Ural Federal University, Ekaterinburg 620000, Russian Federation. E-mail: dmitri.alexandrov@usu.ru occupy only $1 \%$ of the area of the ice cover in order to dominate the heat exchange from the ocean to the atmosphere. In the Arctic winter, the relatively warm water in leads is exposed to the cold air above it. As a result, a thin veneer of ice rapidly forms across an exposed lead. After 1 day's growth the ice layer is about $10 \mathrm{~cm}$ deep, which is still thin compared with the surrounding ice, which is typically $1-2 \mathrm{~m}$ thick. The field observations show that the heat loss through leads can be up to $300 \mathrm{Wm}^{-2}$, or 15 times that from the surrounding ice (The LeadEx Group, 1993). Although leads occupy less than $10 \%$ of the surface area, they are responsible for roughly half of the total oceanic heat loss (The LeADEx Group, 1993). It is known that sea ice growth can be sufficiently fast, so that its depth 8-10 cm can be attained in the first $24 \mathrm{~h}$ (Wettlaufer, Worster and Huppert, 2000; Perovich and Richter-Menge, 2000). A rapid growth of such young sea ice produces the greatest heat flux, so the role of brine drainage on the phase evolution is significant in determining the overall heat budget (see, among others, WetTlaufer, Worster and HupPert, 1997; Alexandrov and Malygin, 2011a). Since the seasonal difference in sea ice coverage is about $12 \times 10^{6} \mathrm{~km}^{2}$ in the Arctic and $14 \times 10^{6} \mathrm{~km}^{2}$ in the Antarctic (see, among others, the NSIDC website http://nsidc.org), the dynamics of the brine rejection processes have a substantial impact on the stability of the oceanic mixed layer and the flux of fresh water into the North Atlantic Ocean, indicating that sea ice export is an important control in convective stability (RAHMSTORF, 1995).

Another mechanism of ice growth during the spring-summer period may be mentioned. When the air temperature becomes above $0{ }^{\circ} \mathrm{C}$ the sea iceatmosphere interface undergoes ablation. As a result, a considerable fraction of meltwater gathers in surface puddles thereby reducing the surface albedo. 
This meltwater percolates into the ice matrix, leading to a strong reduction in the surface salinities. Then it can be retained under thin ice in bottom depressions forming so-called under-ice melt ponds (HANSON, 1965). This low-salinity water comes into contact with seawater, each at or close to their respective freezing points. Laboratory experiments carried out by Martin and KaufFMAn (1974) demonstrate that freezing of under-ice melt ponds is caused due to the double-diffusion mechanism at the interface between freshwater and seawater. Initially, ice platelet crystals appear in the contact zone and then their lateral growth leads to the formation of solid ice cover along the entire freshwater-seawater interface. This underwater ice is called a false bottom (HANSON, 1965; Martin and KaUfFMAn, 1974; EicKEN, 1994; EICKEN et al., 2002). When this underwater ice sheet appears, it freezes upwards due to bottom ablation (see, among others, Hanson, 1965; MARTIN and Kauffman, 1974). The field experiment SHEBA (Surface Heat Budget of the Arctic Ocean) demonstrated that approximately $15 \%$ of a total of more than 100 mass-balance gauges developed false bottoms during the ablation season (EICKEN et al., 2002; Perovich et al., 2003). In accordance with estimates presented by EICKEN (1994) on the study of ice structures in a vast Arctic region, eight ice samples of the 52 total collected for measurements in different locations contained a false bottom. This gives an estimate of approximately $5 \%$ coverage of the ice by the false bottoms assuming that the mean age of the ice is 3 years (EICKEN, 1994; Notz et al., 2003). On the other hand, the traces of false bottoms were found in 22 ice samples of the 57 total investigated in the Beaufort Sea (JEFFriEs et al., 1995). This represents an estimate of approximately $10 \%$ coverage of the ice by the false bottoms assuming that the age of the ice is 4 years. It has been found experimentally that under-floe melt ponds and false bottoms covered half of the flow bottom of the drifting station "Charlie" (HANson, 1965). According to the information available to the authors, this estimate is the upper limit of the ice coverage by false bottoms. As is noted by WADHAMs (1988) on the basis of sonar data, the formation of fresh water reservoirs under the ice cover should be a widely spread phenomenon in the Arctic. NANSEN (1897) from his observations in the Beaufort
Sea noted that the heat transfer from the trapped fresh water, with a temperature of $0{ }^{\circ} \mathrm{C}$, to the arctic sea water, with a temperature of $-1.6{ }^{\circ} \mathrm{C}$, is the only source of ice accretion during the polar summer.

A general global warming trend has made the ice growth processes more sensitive to natural fluctuations in atmospheric and oceanic forcing (Perovich and Richter-Menge, 2009). The present study is devoted to the influence of natural noises induced by different external fluctuations in the ocean and in the atmosphere on the evolution of sea ice growing from above (from the cold atmosphere) and from below (due to the growth of false bottoms).

\section{Sea Ice Growth with a Mushy Layer}

The polar expeditions at the end of the nineteenth century put forward a number of important problems on modeling the processes of ice freezing and their influence on the heat exchange between the ocean and Earth's atmosphere. The first mathematical models describing such processes were developed at the end of the nineteenth century in the pioneering works by STEFAN (1889a, b, 1891). In these papers, he suggests a simple thermal formulation of the problem: heat conductivity equations are satisfied in the solid and liquid phases, while the equality of the temperatures in both phases and the heat balance condition are satisfied at the phase transition boundary. This formulation reflects the main physical behavior of the phase transition: the latent heat releases during the water's freezing. His approach only roughly describes the crystallization process because it does not take into account the ocean salinity. The account for the salt displacement by the growing ice and its diffusion in the liquid within Stefan's thermal problem slightly improves the situation (Buyevich et al., 2001). However, both the purely thermal and thermal diffusion model with a planar front poorly describe the data of different laboratory and field observations [see, among others, HupPert and Worster, (1985), Worster (1986), Alexandrov and Malygin (2006a)]. This is because the phase transition in nature occurs not in a narrow layer approximated by a planar front but in an extended area where the solid and liquid phases are mixed (e.g. 
slush ice). Such a supercooled region originates due to the effect of constitutional supercooling appearing ahead of the phase transition boundary (Ivantsov, 1951). This supercooling arises when the salinity gradient exceeds its temperature analog at the solidliquid interface. As a result, the solid phase in the form of dendrite-like structures grows into the supercooled liquid and creates a region of the mixed state-a mushy or two-phase layer. The mathematical model of mushy layer crystallization was developed a few decades ago by HiLls et al. (1983) and Fowler (1985). However, owing to the complex nonlinear formulation, this moving boundary problem was solved analytically only several years ago. Namely, a method of solving this problem in strongly nonstationary conditions (caused by temperature oscillations in the cold atmosphere) in the case of an isothermal ocean was developed by ALEXANDrov and MALygin (2006a) and AleXANDrov et al. (2006). Then this theory of ice crystallization with a mushy layer was developed for the case of turbulent flows in the ocean (AlEXANDrov et al., 2010). Moreover, the growth of ice with a false bottom mushy layer is recently described analytically by ALEXANDrov and Nizovtseva (2008) and Alexandrov and Malygin (2011b).

All of these models will be used in the present study to describe the influence of external stochastic fluctuations on the sea ice dynamics with a mushy layer. Let us note the main physical hypotheses used in our previous theories to obtain analytical solutions of corresponding models describing freezing processes from above and from below (the final solutions of these models will be analyzed in Sects. 3 and 4 to study the role of stochastic fluctuations of corresponding parameters on the nonlinear dynamics of crystallization processes). The phase transition zone represents a quasi-equilibrium mushy layer (HILls et al., 1983; BUYEVICH et al., 2001). Since a relaxation time of the temperature field is far less than characteristic times of the process (a relaxation time of the salinity field or a characteristic time of the motion of the phase transition boundary), the temperature field in the mushy layer (false bottom) is considered as a linear function of the spatial coordinate. A more detailed explanation of the final analytical expressions in Sects. 3 and 4 can be found in our previous studies (AlEXANDRov and Malygin, 2006a, 2011b; Alexandrov et al., 2006, 2010; Alexandrov and NizovTSEVA, 2008).

Let us mention the paper of L'HÉLVÉDER and Houssais (2001) where a significant role of stochastic atmospheric forcing on the sea ice dynamics has been revealed on the basis of simplified heat and mass transfer equations (without a mushy layer) and equivalent Markov model constructed by linearizing the ice growth rate equations. The present paper develops this theme further by considering non-linear heat and mass transfer processes in the sea ice mushy layer with corresponding non-linear contributions of the external noises.

\section{Freezing from Above}

Let us consider the process of sea ice freezing with a phase transition region illustrated in Fig. 1. As mentioned above, the theory and methods of analytical solutions describing this nonstationary process in the case of arbitrary temperature oscillations in the atmosphere were developed by AlEXANDRov and Malygin (2006a), Alexandrov et al. (2006) and AleXANDrov et al. (2010). These papers demonstrate that the ice thickness $b$ (the mushy layer-ocean boundary) as a function of crystallization time $t$ is determined by the following expression

\section{Atmosphere}

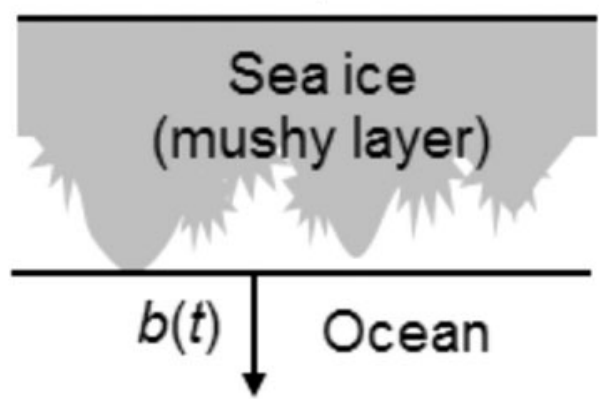

Figure 1

A schematic diagram of the freezing process from above. A region where liquid (white) and solid (grey) co-exist, mushy layer, is between the ocean and the atmosphere 


$$
\begin{aligned}
& b(t)=\sqrt{\frac{2}{I}\left(T_{w} t-\int_{0}^{t} T_{a t}(\alpha) \mathrm{d} \alpha\right)^{+}} \\
& I=A\left[1-\frac{D_{w} A}{T_{w}}\left(\frac{L_{V} D_{w}}{k_{i} T_{w}}+1-\frac{k_{w}}{k_{i}}\right)\left(\varphi_{b}-1\right)\right]
\end{aligned}
$$

Here $\quad A=L_{V} \varphi_{b} / \Phi, \Phi=k_{i} \varphi_{b}+k_{w}\left(1-\varphi_{b}\right), L_{V}$ -is the latent heat parameter, $D_{w}$ - the diffusion coefficient of salt in water, $\varphi_{b}$-the solid fraction at the mushy layer-ocean boundary $b, T_{w}$-the temperature in the isothermal ocean near this boundary, $k_{i}$ and $k_{w}$ - the thermal conductivities of the sea ice and salt water, $T_{a t}(t)$ - the atmospheric temperature (temperature at the boundary between the sea ice and the atmosphere). The sign "plus" is introduced as $x^{+}=x$ at $x \geq 0$ and $x^{+}=0$ at $x<0$; it reflects the fact that the under-root expression in (1) is always positive. Note that the limiting case $\varphi_{b} \rightarrow 1$ describes the frontal solution (AlEXANDrov and MALYGin, 2006a; Alexandrov et al., 2006). Expression (1) describes the field observations (The LEADEx Group, 1993; Notz et al., 2003) and represents the analytical solution of the Stefan-type problem with a mushy layer, which freezes into the isothermal ocean due to an arbitrary temperature drop with time in the atmosphere. Also, this expression shows that the ice (mushy layer) boundary solidifies in the self-similar manner $b(t)=$ $\sqrt{2 \delta t / I}$ if the atmospheric temperature differs in a constant from the oceanic temperature, that is, if $T_{a t}(t)=T_{w}-\delta(\delta>0)$. This regime describes the final (later) stages of the crystallization process (ALEXANDROV and MALYGIN, 2006b) when $b(t) \sim \sqrt{t}$. Note that expression (1) is analytically derived in the case of linear temperature in the sea ice. For detailed explanation, see the theory (AlEXANDrov and MALYGIN, 2006a; AlEXANDrov et al., 2006, 2010) based on the field observations (The LEADEX Group, 1993; Notz et al., 2003).

Let us now consider how possible fluctuations in the atmospheric temperature influence on the ice growth dynamics. First, we study the simplest case describing periodic fluctuations of the atmospheric temperature $T_{a t}(t)=T_{w}+\delta \cos \omega t$ about its averaged value $T_{w}$, which coincides with the sea water temperature. Here $\delta$ and $\omega$ stand for the amplitude and frequency of fluctuations. In other words, this regime corresponds to a constant temperature in the system (in the atmosphere, sea ice and ocean) equal to $T_{w}$ in the absence of fluctuations. In this case, expression (1) shows that the sea ice (mushy layer) thickness oscillates as $b(t)=\sqrt{2 \delta(-\sin \omega t)^{+} / \omega I}$ (this regime is demonstrated in Fig. 2). Let us note that the mushy layer thickness increases as the frequency of oscillations decreases due to the effect of sea ice inertness.

It is well known that transient air motions, turbulence and inhomogeneous clouds lead to stochastic fluctuations in the air temperature on the sea ice surface (see, among others, Notz et al., 2005). Such randomized impacts can change drastically the dynamics of nonlinear systems similar to the sea ice. As it is known in physics, there is a broad variety of natural phenomena and processes connected with different type of noises. So, for example, the stochastic resonance and bifurcations, noise-induced transitions and chaos may be mentioned among others (Horsthemke and Lefever, 1984; Anishchenko et al., 2007; BashKirTSEva and RyAshKo, 2009; BASHKIRTSEVA et al., 2012). We demonstrate below how stochastic fluctuations in the main external parameters influence on the sea ice dynamics.

Let us generate random fluctuations in the atmospheric temperature $T_{\text {at }}(t)=T_{w}+\varepsilon \xi(t)$ by means of the Ornstein-Uhlenbeck process $\xi(t)$, which is determined from the following Langevin equation (GARDINER, 2009)

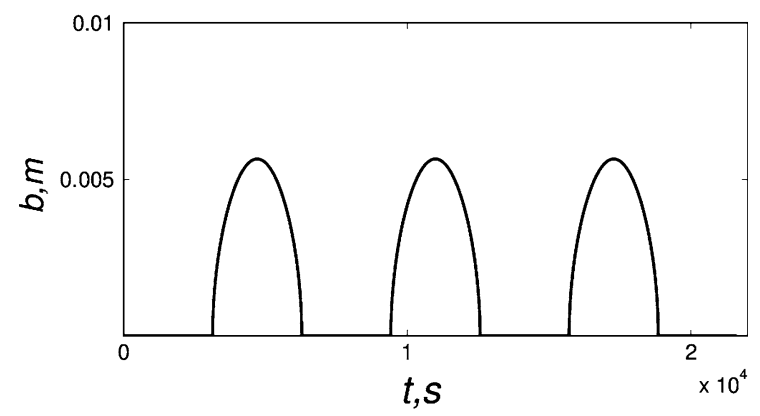

Figure 2

The sea ice (mushy layer) thickness accordingly to expression (1) in the case of periodic oscillations of temperature in the atmosphere. Physical parameters used for calculations are given by Alexandrov and Malygin (2006a) and Alexandrov et al. (2006), $\delta=2, \omega=0.001$ 


$$
\frac{d \xi}{d t}=-p \xi+\sqrt{2 p} \frac{d w}{d t}
$$

where $w(t)$ and $\varepsilon$ stand for the standard Wiener process and intensity of stochastic fluctuations of the atmospheric temperature respectively. Note that the mean value function $E$ for the Wiener process possesses the following properties $E(w(t)-w(s))=0$ and $E(w(t)-w(s))^{2}=|t-s|$. The steady-state solution $\xi(t)$ of Eq. (2) is characterized by the mean value function $E(\xi(t))=0$, stochastic variance $E(\xi(t))^{2}=$ 1 and autocovariance function $\operatorname{cov}(\xi(t), \xi(t+\tau))=$ $\exp (-p \tau)$, where parameter $p$ determines different covariance structures. In other words, by varying this parameter one can change the process covariance, that is a small value of $p$ corresponds to slow random variations in the atmospheric temperature. As this value grows the frequency of $\xi(t)$ increases and the limiting case $p \rightarrow \infty$ shifts the random process to the white noise.

Figure 3 illustrates a sample path of the atmospheric temperature $T_{a t}(t)=T_{w}+\varepsilon \xi(t)$ for $T_{w}=$ $-2^{\circ} \mathrm{C}, \varepsilon=1$ and $p=0.01$ (for these parameters, time series of $T_{a t}(t)$ looks quite realistic). Note that temperature oscillations $T_{a t}(t)$ lie within the confidence interval $\left(T_{w}-2{ }^{\circ} \mathrm{C}, T_{w}+2{ }^{\circ} \mathrm{C}\right)$ in accordance with the two sigma rule with a 0.95 probability. Each stochastic sample path therewith determines a certain ice thickness $b(t)$ accordingly to expression (1). Let us especially emphasize that a natural deterministic parameter of the process under consideration can be expressed in terms of the assembly average $\left\langle T_{a t}(t)\right\rangle=$ $T_{w}$, which is independent of parameters $p$ and $\varepsilon$, whereas the mushy layer thickness $\langle b(t)\rangle$ is highly dependent upon their variation (Figs. 4, 5).

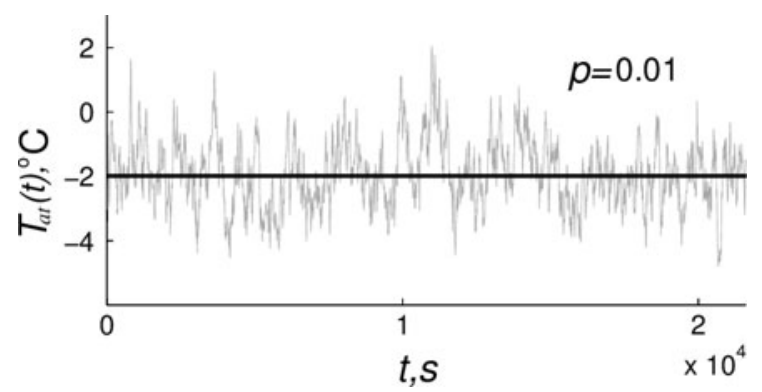

Figure 3

The sample path of atmospheric temperature in the case of stochastic fluctuation about its mean value $\left\langle T_{a t}(t)\right\rangle=-2{ }^{\circ} \mathrm{C}$.
Figures 3, 4, 5 plotted for the case when the mean value of the atmospheric temperature coincides with the constant temperature in the ocean $\left(\left\langle T_{\text {at }}(t)\right\rangle=T_{w}\right)$ show that stochastic fluctuations about this average temperature induce the sea ice (mushy layer) freezing. This is explained by interaction of the crystallization and diffusion processes. If the instant temperature in the atmosphere falls below the phase transition temperature (at a fixed salinity), the salt water freezes. As this takes place, the growing ice crystals will displace all salt into the liquid. If the atmospheric temperature becomes greater than the melting temperature of pure water (intense fluctuations), the melting process is possible. However, as the system temperature is below zero in a great part of time and intense fluctuations are rare (Fig. 3), the sea ice has no tendency to melt. This is caused by different phase transition temperatures of the salt water freezing (e.g. $2{ }^{\circ} \mathrm{C}$ ) and the sea ice melting (e.g. $0{ }^{\circ} \mathrm{C}$ ). As a result, the phase transition boundary migrates downwards due to stochastic fluctuations about a constant temperature in the whole system. Note that the sea ice freezes faster when the intensity of stochastic fluctuations $\varepsilon$ increases and $p$ decreases (Figs. 4, 5).

Let us now consider the case when a stochastically fluctuating atmospheric temperature lies below than the ocean temperature, i.e. $T_{\text {at }}(t)=T_{w}-\Delta+$ $\varepsilon \xi(t)$, where $\Delta$ is a constant. Figure 6 illustrates some sample paths for the mushy layer thickness $b(t)$. It is easily seen that fluctuating sample paths deviate irregularly from the mushy layer thickness without

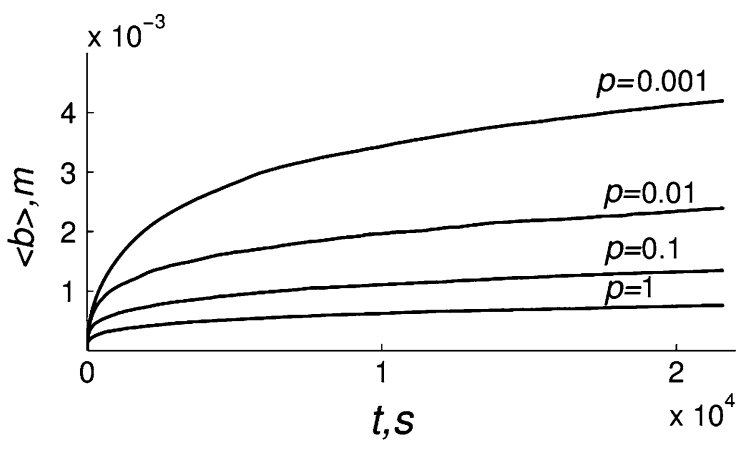

Figure 4

The sea ice (mushy layer) thickness accordingly to expression (1) in the case of stochastic oscillations of temperature in the atmosphere, $\varepsilon=1$ 


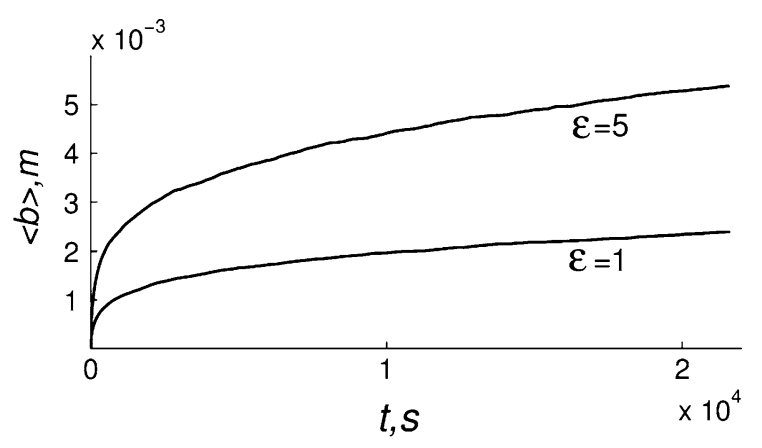

Figure 5

The sea ice (mushy layer) thickness accordingly to expression (1) in the case of stochastic oscillations of temperature in the atmosphere, $p=0.01$

fluctuations $(\varepsilon=0)$. In addition, maximal deviations occur at the initial stage of mushy layer freezing, whereas all oscillations are decreasing when crystallization time increases. In other words, the initial crystallization stage is rather sensitive to stochastic temperature fluctuations in the atmosphere which are responsible for the formation of young sea ice in the form of a mushy layer.

Now let us pay attention to a more realistic casedescribing diurnal temperature oscillations accompanied by occasional fluctuations when the atmospheric temperature behaves as $T_{\text {at }}(t)=T_{w}+$ $\delta \cos \omega t+\varepsilon \xi(t)$. This freezing regime is illustrated in Fig. 7. One can readily see that periodic growth stages (freezing and melting similar to Fig. 2) occur in the absence of stochastic fluctuations $(\varepsilon=0)$. As this takes place, the sea ice (mushy layer) thickness increases more rapidly with increasing intensity of stochastic fluctuations. This behavior is explained by

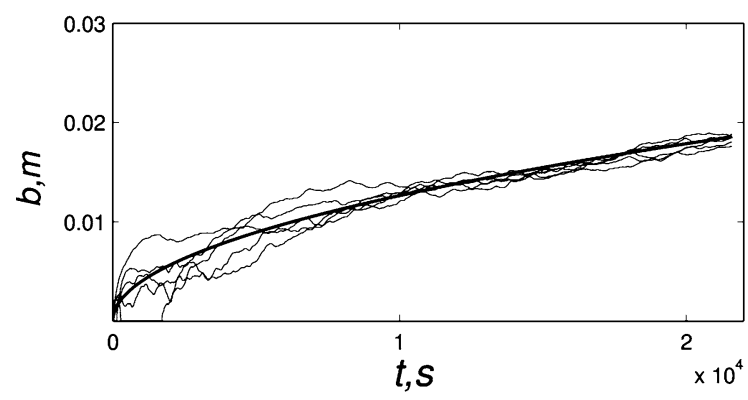

Figure 6

Sample paths of the sea ice (mushy layer) thickness at $T_{w}=$ $-2{ }^{\circ} \mathrm{C}, p=0.01, \varepsilon=2$ and $\Delta=1$. The solid line represents the mushy layer thickness without fluctuations $(\varepsilon=0)$ interaction of the crystallization and diffusion processes discussed above. Note that the same nondeterministic behavior of ice thickness has been discussed by Timmermann et al. (2002) on the basis of experimental data and numerical simulations.

\section{Freezing from Below}

Another interesting example of sea ice growth is the evolution of false bottoms (Fig. 8). The main fluctuating parameter in this freezing process is the friction velocity $u_{*}$ (UUSIKIVI et al., 2006; HAYES and Morison, 2008). This velocity characterizes the thermal and salinity turbulent fluxes at the false bottom-ocean boundary

$$
\left\langle u^{\prime} T^{\prime}\right\rangle=\alpha_{h} u_{*}\left(T_{\infty}-T_{f}\right),\left\langle u^{\prime} S^{\prime}\right\rangle=\alpha_{s} u_{*}\left(S_{\infty}-S_{f}\right)
$$

where $\alpha_{h}$ and $\alpha_{s}$ are the turbulent transfer coefficients for heat and salt, $T_{\infty}-T_{f}$ and $S_{\infty}-S_{f}$ are the temperature and salinity differences deep in the ocean (temperature $T_{\infty}$ and salinity $S_{\infty}$ ) and at the false bottom-ocean boundary (temperature $T_{f}$ and salinity $\left.S_{f}\right)$.

Let us study the role of stochastic fluctuations on the false bottom dynamics on the basis of analytical solutions previously derived by ALEXANDROV and Nizovtseva (2008) and Alexandrov and Malygin (2011b). In accordance with their theory the false bottom thickness $h(t)=g(t)-f(t)$ representing a mushy layer is determined from the following system of differential equations

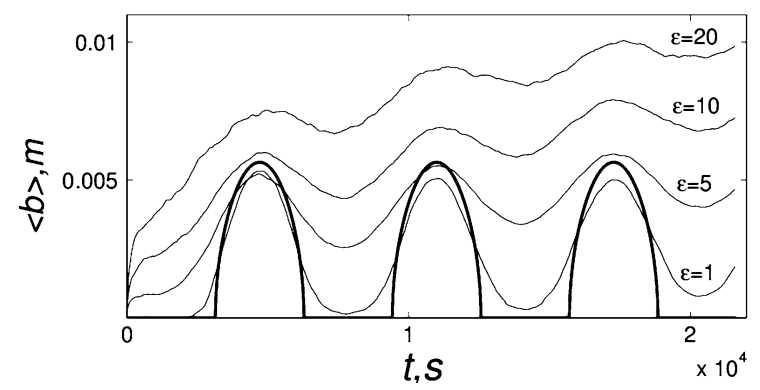

Figure 7

The sea ice (mushy layer) thickness accordingly to expression (1) in the case of periodic and stochastic oscillations of temperature in the atmosphere, $\delta=2, \omega=0.001$. The solid line represents the mushy layer thickness without stochastic fluctuations $(\varepsilon=0)$ 


\section{Atmosphere}

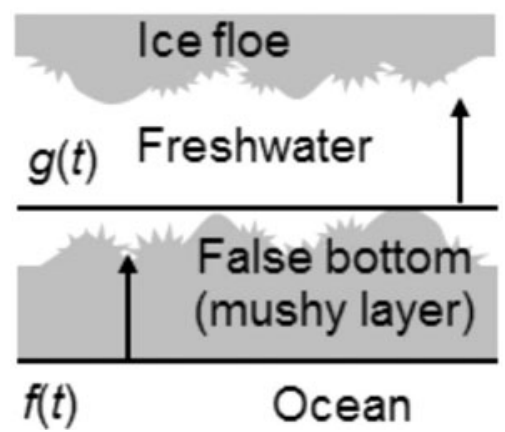

Figure 8

A schematic diagram of the freezing process from below with a false bottom. The solid and liquid phases in the mushy layer of the false bottom are shown by grey and white regions respectively

$$
\begin{aligned}
\frac{d h}{d t} & =\frac{P_{1}\left(T_{g}(t)\right)}{h(t)}+P_{2}\left(T_{g}(t), t\right), h(t) \\
& =-\frac{\left(k_{i} \varphi_{f}+k_{w}\left(1-\varphi_{f}\right)\right)\left(T_{g}-T_{f}\right) T_{f}}{\alpha_{s} u_{*} L_{V}\left(T_{f}+m S_{\infty}\right)+\alpha_{h} \rho_{w} c_{w} u_{*}\left(T_{\infty}-T_{f}\right) T_{f}}
\end{aligned}
$$

where

$$
\begin{aligned}
& P_{1}=\frac{\left(k_{i} \varphi_{g}+k_{w}\left(1-\varphi_{g}\right)\right)\left(T_{g}-T_{f}\right)}{L_{V} \varphi_{g}}, \\
& P_{2}=\frac{\alpha_{s} u_{*}\left(T_{f}+m S_{\infty}\right)}{T_{f} \varphi_{f}}, \varphi_{g}(t)=\frac{K T_{g}(t)}{(K-1) T_{g}(t)-T_{p}} \\
& T_{f}\left(T_{g}(t)\right)=\frac{T_{g}^{2}(t)+T_{g}(t) T_{p}}{\left(\varphi_{f}-1\right)\left[(K-1) T_{g}(t)-T_{p}\right]}, \\
& T_{p}=\frac{D_{w} L_{V}}{k_{i}}, K=\frac{k_{w}}{k_{i}}
\end{aligned}
$$

Here $m$ is the liquidus slope, $T_{g}, \varphi_{g}$ and $T_{f}, \varphi_{f}$ are the temperatures and solid fractions at the phase transition boundaries $g(t)$ and $f(t)$. These expressions represent a standard Cauchy problem for the numerical calculation of temperature $T_{g}(t)(h(0)$ is a given value) after substitution of $h(t)$ and $T_{f}\left(T_{g}(t)\right)$ in the first Eq. (3) whereupon the false bottom thickness becomes known from the second Eq. (3). Note that analytic solutions of this problem are obtained in the case of linear temperature profile in the false bottom previously observed by MARTIN and KAUFFMAN (1974) in their laboratory experiments. For detailed explanations of used physical hypotheses see also original papers (AleXANDRov and Nizovtseva, 2008; Alexandrov and Malygin, 2011b) devoted to the theory of false bottom evolution.

Our previous studies based on the field experiments (AIDJEX and SHEBA) demonstrate a key role of the friction velocity on nonlinear dynamics of the false bottom ice evolution (AlEXANDrov and Nizovtseva, 2008; Alexandrov and Malygin, 2011b). So, for example, the false bottom thickness typically increasing with time becomes a decreasing function when $u_{*}$ increases abruptly. The physical reason is that a storm came through, which substantially increased the friction velocity (see, among others, Fig. 5 illustrated by Alexandrov and Nizovtseva, 2008). As a result, the salt flux from the ocean to the ice increases, and in its turn a rapid ablation of the false bottom occurs. Taking into account an important role of the friction velocity oscillations on the false bottom growth we model below their natural fluctuations by means of the known stochastic methods.

Let us model the random process of friction velocity fluctuations by means of the following stochastic differential equation

$$
\frac{d^{2} v}{d t^{2}}+k \frac{d v}{d t}+l v=\varepsilon \frac{d w_{1}}{d t}
$$

with nonlinear positively defined friction velocity

$$
u_{*}= \begin{cases}v, & v \geq q \\ q \exp \left(\frac{v-q}{q}\right), & v<q\end{cases}
$$

Here $w_{1}(t)$ is a standard Gaussian process with mean values $\left\langle w_{1}(t)-w_{1}(\tau)\right\rangle=0$ and $\left\langle\left(w_{1}(t)-\right.\right.$ $\left.\left.w_{1}(\tau)\right)^{2}\right\rangle=t-\tau$, and $k, l, \varepsilon$ and $q$ represent the process parameters. Such choice of the dynamic model (4) and (5) for the friction velocity fluctuations is dictated by the following. It can easily be shown that the differential equation for $T_{g}(t)$ and, as a consequence, expression (3) for $h(t)$ include the friction velocity derivatives $\frac{d u_{*}}{d t}$. If the random fluctuations will be modeled by means of a first-order differential equation similar to equation (2), the derivative $\frac{d u_{*}}{d t}$ will have the unlimited dispersion. To avoid this, we model the friction velocity fluctuations 
by means of a simple second-order differential Eq. (4) of stochastically forced linear oscillator with damping coefficient $k$ and stiffness $l$ (ANISHCHENKO et al., 2007; GARDINER, 2009). The nonlinear transformation (5) represents one of possible models of a smooth and positively defined function $u_{*}$ with a small normalizing coefficient $q$. Note that the used extension of the friction velocity $q \exp \left(\frac{v-q}{q}\right)$ is required for its smoothing.

Estimating the natural fluctuations of the friction velocity (Uusikivi et al., 2006; HAyes and Morison, 2008), we demonstrate its sample path in Fig. 9. Corresponding stochastic paths of fluctuations of the false bottom thickness and its mean value are shown in Fig. 10. A key result is that random fluctuations of the friction velocity produced by turbulent motions in the ocean substantially reduce the false bottom thickness (approximately $30 \%$ reduction in the false bottom thickness after the 5-day growth). Physically, this is caused by the fact that friction velocity fluctuations produce corresponding fluctuations of temperature $T_{g}$, whose averaged value is about $0{ }^{\circ} \mathrm{C}$ (Martin and Kauffman, 1974). Ice crystals in the false bottom at the phase transition boundary $g(t)$ (Fig. 8) should undergo melting with a rise in $T_{g}$. Salt water at this boundary, on the other hand, has a negative freezing temperature (dependent of salinity) preventing its crystallization with decreasing $T_{g}$.

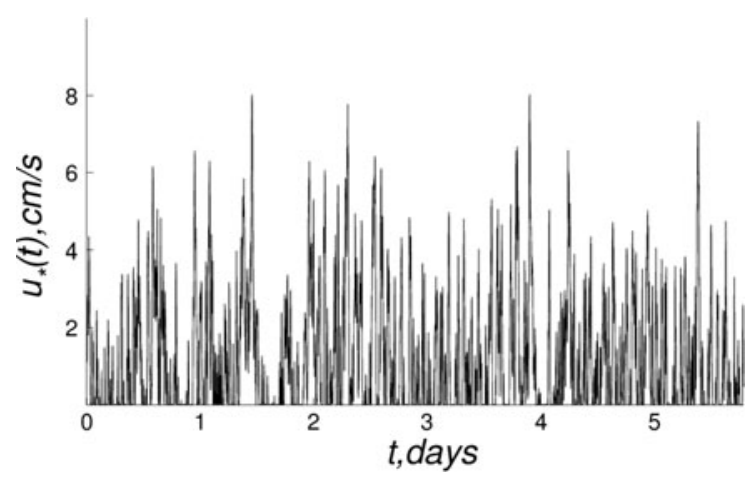

Figure 9

A sample path of the friction velocity with the mean value $\left\langle u_{*}(t)\right\rangle=1.2 \mathrm{~cm} \mathrm{~s}^{-1}$. The following set of model parameters $k=$ $10^{-2}, l=10^{-5}, \varepsilon=10^{-3}, q=10^{-1}$ is fixed. Physical parameters used for calculations are given by AleXANDrov and Nizovtseva (2008) and AleXandrov and MaLygin (2011b)

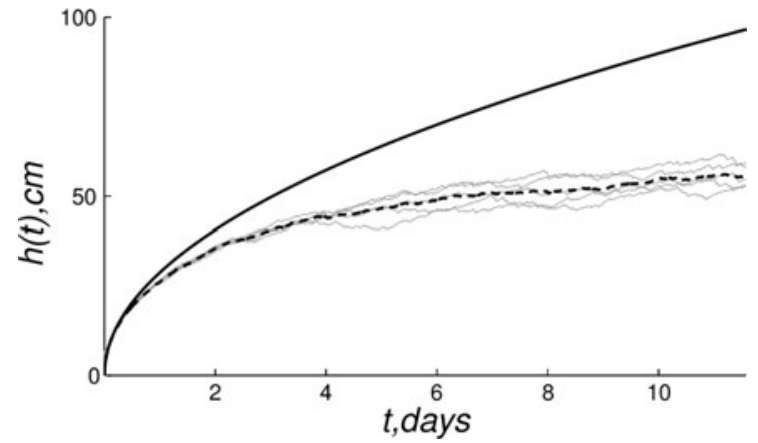

Figure 10

Sample paths of the false bottom thickness (grey lines) and its mean value (dashed line) accordingly to expressions (3) in the case of stochastic oscillations of the friction velocity. The solid line represents the false bottom thickness without stochastic fluctuations

\section{Concluding Remarks}

It is well-known that ice freezing makes an important contribution to the heat budget between the ocean and the atmosphere. So, for example, approximately half of the surface heat flux released into the atmosphere during an arctic winter is caused by the latent crystallization heat. The heat exchange processes between the ocean and the atmosphere play an important role in the air mass motion and influence the formation of the weather conditions. In order to take into account the influence of these processes in the atmospheric dynamics it is necessary to estimate the role of different stochastic processes met in the ocean and the atmosphere on the ice growth processes. A significant part of the sea ice is formed by freezing from above in the cold winter season. In the spring-summer period, the false bottom freezing processes from below represent the only source of ice accretion. Taking into consideration these two main sources of ice growth we study the influence of natural noises on the ice formation.

Our analysis based on analytical theories of ice growth from above and from below as well as on the stochastic differential models (2) and (4) used for the noise generation demonstrate that the nonlinear dynamics of ice growth is highly dependent on the variance of stochastic fluctuations of the atmospheric temperature and the friction velocity. Namely, even in the case of equal temperatures in the atmosphere and in the ocean, temperature dispersion in the 
atmosphere caused by occasional processes (noises) results in sea ice freezing. In addition, the rate of ice growth increases with increasing temperature variance in the atmosphere and attains several centimeters per day. On the other hand, stochastic fluctuations caused by turbulent flows in the ocean reduce the sea ice thickness produced by false bottom evolution processes. Our estimates show that this reduction in the order of magnitude can be up to $10 \mathrm{~cm}$ per several days.

What this means is the ice freeze-on rate is essentially different in the case of consideration of natural noises. The freezing process from below occurs, in particular, under ice shelves, where freshwater glacial run-off at a temperature very near $0{ }^{\circ} \mathrm{C}$ accumulates behind the ice shelf until the fresh water flows out beneath the shelf. Another interesting example is that the East Antarctic ice sheet grows, in particular, by freezing from the base and in some places, up to half of the ice thickness freezes from below (Bell et al., 2011). Our model results, in particular, show that these ice growth processes from below will be decelerated by natural fluctuations of the oceanic velocity.

\section{Acknowledgments}

We are grateful for partial support from the Federal Target Program "Scientific and scientific-pedagogical personnel of innovative Russia" in 2009-2013 and the Russian Foundation for Basic Research (Project No. 11-01-00137).

\section{REFERENCES}

AagARD, K., and CARMACK, E. (1994). The arctic ocean and climate: a perspective, in The Polar Regions and Their Role in Shaping the Global Environment, ed by Johannessen, O.M., Muench, R.D., and Overland, J.E. Geophys. Monogr. Ser. 85, AGU, pp. 5-20.

Alexandrov, D.V., and MAlygin, A.P. (2006a). Analytical description of seawater crystallization in ice fissures and their influence on heat exchange between the ocean and the atmosphere. Dokl. Earth Sci. 411A, 1407-1411.

Alexandrov, D.V., and Malygin, A.P. (2006b). Self-similar solidification of an alloy from a cooled boundary. Int. J. Heat Mass Transf. 49, 763-769.

Alexandrov, D.V., Malygin, A.P., and Alexandrova, I.V. (2006). Solidification of leads: approximate solutions of non-linear problem. Ann. Glaciol. 44, 118-122.
Alexandrov, D.V., and Malygin, A.P. (2011a). Convective instability of directional crystallization in a forced flow: The role of brine channels in a mushy layer on nonlinear dynamics of binary systems. Int. J. Heat Mass Transf. 54, 1144-1149.

Alexandrov, D.V., and Malygin, A.P. (2011b). Nonlinear dynamics of phase transitions during seawater freezing with a false bottom formation. Oceanology 51, 940-948.

Alexandrov, D.V., and Nizovtseva, I.G. (2008). To the theory of underwater ice evolution, or nonlinear dynamics of "false bottoms”. Int. J. Heat Mass Transf. 51, 5204-5208.

Alexandrov, D.V., Nizovtseva, I.G., Lee, D., and Huang, H.-N. (2010). Solidification from a cooled boundary with a mushy layer under conditions of nonturbulent and turbulent heat and mass transfer in the ocean. Int. J. Fluid Mech. Res. 37, 1-14.

Anishchenko, V.S., Astakhov, V.V., Neiman, A.B., Vadivasova, T.E., and Schimansky-Geier, L., Nonlinear Dynamics of Chaotic and Stochastic Systems. Tutorial and Modern Development (Springer-Verlag, Berlin/Heidelberg 2007).

Badgley, F.J. (1966). Heat budget at the surface of the Arctic Ocean, in Proceedings of Symposium on the Arctic Heat Budget and Atmospheric Circulation, ed by Fletcher, J.O. Santa Monica, CA, Rand Corporation, pp. 267-277.

Bashirtstseva, I., and Ryashro, L. (2009). Constructive analysis of noise-induced transitions for coexisting periodic attractors of Lorenz model. Phys. Rev. E 79, 041106-041114.

Bashirirtseva, I., Chen, G., and Ryashko, L. (2012). Analysis of noise-induced transitions from regular to chaotic oscillations in the Chen system. Chaos 22, 033104.

BELL, R.E. et al. (2011). Widespread persistent thickening of the East Antarctic ice sheet by freezing from the base. Science 331, 1592-1595.

Buyevich, Yu. A., Alexandrov, D.V., Mansurov, V.V., Macrokinetics of crystallization (Begell House, New York-Wallingford 2001) 183.

EICKEN, H. (1994). Structure of under-ice melt ponds in the central Arctic and their effect on the sea-ice cover. Limnol. Oceanogr. 39, 682-694.

Eicken, H., Krouse, H.R., Kadko, D., and Perovich, D.K. (2002). Tracer studies of pathways and rates of meltwater transport through Arctic summer sea ice. J. Geophys. Res. 107, 8046.

Fowler, A.C. (1985). The formation of freckles in binary alloys. IMA J. Appl. Maths. 35, 159-174.

GARDINER, C., A handbook for the natural and social sciences (Springer, Series in Senergetics 2009) 447.

Hanson, A.M. (1965). Studies of the mass budget of Arctic pack-ice floes. J. Glaciol. 5, 701-709.

HAYes, J., and Morison, J. (2008). Ice-ocean turbulent exchange in the Arctic summer measured by an autonomous underwater vehicle. Limnol. Oceanogr. 53, 2287-2308.

Hills, R.N., Loper, D.E., and RoberTs, P.H. (1983). A thermodynamically consistent model of a mushy zone. Q. J. Appl. Math. $36,505-539$.

Horsthemke, W., and Lefever, R., Noise-Induced Transitions (Springer, Berlin 1984).

HupPert, H.E., and Worster, M.G. (1985). Dynamic solidification of a binary melt. Nature 314, 703-707.

Ivantsov, G.P. (1951). Diffusional supercooling during crystallization of a binary alloy. Dokl. Akad. Nauk SSSR 81, 179-182.

Jeffries, M.O., Schwartz, K., Morris, K., Veazey, A.D., Krouse, H.R., and GushinG, S. (1995). Evidence for platelet ice accretion 
in Arctic sea ice development. J. Geophys. Res. 100, $10,905-10,914$.

L'HÉLVÉDER, B., and HousSAIS, M.-N. (2001). Investigating the variability of the Arctic sea ice thickness in response to a stochastic thermodynamic atmospheric forcing. Climate Dynamics, $17,107-125$.

Martin, S., and Kauffman, P. (1974). The evolution of under-ice melt ponds. J. Fluid Mech. 64, 507-527.

Nansen, F., Farthest North (Harper and Brothers Rublishers, New York 1897) 714.

Notz, D., McPhee, M.G., Worster, M.G., MAYкut, G.A., SCHLÜNZEN, K.H., and Eicken, H. (2003). Impact of underwaterice evolution on Arctic summer sea ice. J. Geophys. Res. 108, 3223.

Notz, D., Wettlaufer, J.S., Worster, M.G. (2005). A nondistructive method for measuring the salinity and solid fraction of growing sea ice in situ. J. Glaciol. 51, 159-166.

Perovich, D.K., Grenfell, T.C., Richter-Menge, J.A., Light, B., TUCKER, W.B., and EICKEN, H. (2003). Thin and thinner: ice mass balance measurements during SHEBA. J. Geophys. Res. 108, 8050.

Perovich, D.K., and Richter-Menge, J.A. (2000). Ice growth and solar heating in springtime leads. J. Geophys. Res. 105, 6541-6548.

Perovich, D.K., and Richter-Menge, J.A. (2009). Loss of sea ice in the Arctic. Annu. Rev. Marine Sci. 1, 417-441.

RAHMStoRf, S. (1995). Bifurcations of the Atlantic thermohaline circulation in response to changes in the hydrological cycle. Nature 378, 145-149.

STEFAN, J. (1889a). Über einige Probleme der Theorie der Wärmeleitung. Sitzungsberichte de Mathematisch-Naturawissenschaftlichen
Classe der Kaiserlichen, Akademie der Wissenschaften 98(2a), 473-484.

STEFAn, J. (1889b). Über die Theorie der Eisbildung, insbesondere über die Eisbildung im Polarmeere. Sitzungsberichte de Mathematisch-Naturawissenschaftlichen Classe der Kaiserlichen, Akademie der Wissenschaften 98(2a), 965-983.

STEFAn, J. (1891). Über die Theorie der Eisbildung, insbesondere über die Eisbildung im Polarmeere. Ann. Der Physik u. Chem., Neue Folge 42, 269-286.

The LeadEx Group (1993). The LeadEx experiment. EOS Trans. AGU 74, 393, 396-397.

Timmermann, R., Beckmann, A., and Hellmer, H.H. (2002). Simulations of ice-ocean dynamics in the Weddell Sea 1. Model configuration and validation. J. Geophys. Res. 107, 3024.

Uusikivi, J., Ehn, J., and Granskog, M.A. (2006). Direct measurements of turbulent momentum, heat and salt fluxes under landfast ice in the Baltic Sea. Ann. Glaciol. 44, 42-46.

WadHAms, P. (1988). The underside of Arctic sea ice imaged by sidescan sonar. Nature 333, 161-164.

Wettlaufer, J.S., Worster, M.G., and Huppert, H.E. (1997). Natural convection during solidification of an alloy from above with application to the evolution of sea ice. J. Fluid Mech. 344, 291-316.

Wettlaufer, J.S., Worster, M.G., and Huppert, H.E. (2000). Solidification of leads: theory, experiment, and field observations. J. Geophys. Res. 105, 1123-1134.

Worster, M.G. (1986). Solidification of an alloy from a cooled boundary. J. Fluid. Mech. 167, 481-501. 